\title{
Association of BAK1 single nucleotide polymorphism with a risk for dengue hemorrhagic fever
}

Tran Ngoc Dang ${ }^{1,2}$, Izumi Naka ${ }^{1,3,4}$, Areerat Sa-Ngasang ${ }^{5}$, Surapee Anantapreecha ${ }^{5}$, Nuanjun Wichukchinda ${ }^{5}$, Pathom Sawanpanyalert ${ }^{6}$, Jintana Patarapotikul ${ }^{7}$, Naoyuki Tsuchiya ${ }^{1,3}$ and Jun Ohashi, ${ }^{1,3,4^{*}}$

\begin{abstract}
Background: Dengue hemorrhagic fever (DHF) is a severe life-threatening form of dengue infection. Low platelet count is one of the characteristic clinical manifestations in patients with severe dengue. However, little is known about genetic factors in the host that cause low platelet count in patients with dengue.

Methods: A previous genome-wide association study of hematological and biochemical traits identified single nucleotide polymorphisms (SNPs) associated with low platelet count in healthy subjects. To examine the possible association of these SNPs with DHF, 918 Thai patients with dengue [509 patients with DHF and 409 with dengue fever (DF)] were genotyped for five SNPs: rs5745568 in BAK1, rs6141 in THPO, rs6065 in GP1BA, rs739496 in SH2B3, and rs385893 in RCL1. In addition, rs4804803 in CD209, that has been reported to be associated with dengue infection, was also genotyped to examine if rs4804803 affects the association detected in this study.

Results: The allele frequencies of each SNP were compared between the DHF and DF groups. Among the five SNPs, the $G$ allele of rs 5745568 in BAK1 was significantly associated with a risk for DHF $[P=0.006$ and crude odd ratio $(95 \%$ confidence interval $)=1.32(1.09-1.60)]$. The association of this allele with DHF was also significant in a logistic regression analysis adjusted for age, sex, hospital (i.e., geographic region), immune status (i.e., primary or secondary infection), and virus serotype $[P=0.016$ and adjusted odd ratio (95\% confidence interval) $=1.29$ (1.05-1. 58)]. The result was not influenced by $\operatorname{rs} 4804803[P=0.0167$ and adjusted $\mathrm{OR}(95 \% \mathrm{Cl})=1.29(1.05-1.58)]$. No other SNPs including rs4804803 showed significant association.
\end{abstract}

Conclusions: The low-level constitutive production of platelets caused by the $\mathrm{G}$ allele of rs5745568 seems to increase the risk of bleeding in dengue infection. Our results suggest that BCL-2 homologous antagonist/killer (BAK) protein, encoded by BAK1, plays a crucial role in the pathogenesis of DHF.

\section{Background}

Dengue is a mosquito-borne acute systemic viral infection caused by the dengue virus (DEN) [1]. DEN is a small single-stranded RNA virus consisting of four distinct serotypes, DEN-1 to DEN-4 [2]. The severity of DEN infection ranges from a mild disease, dengue fever (DF), to the severe dengue hemorrhagic fever (DHF), which can culminate in dengue shock syndrome (DSS) and death [3]. The vast majority of severe dengue cases

\footnotetext{
* Correspondence: juno-tky@umin.ac.jp

${ }^{1}$ Graduate School of Comprehensive Human Sciences, University of Tsukuba, Tsukuba, Japan

${ }^{3}$ Faculty of Medicine, University of Tsukuba, Tsukuba, Japan

Full list of author information is available at the end of the article
}

occur in children and are currently a leading cause of hospital admission and death among children in Asia [4]. The transmission has been estimated to occur in up to 124 countries [5], with at least 50 million infected cases per year [6], including 250,000 cases of DHF/DSS [7]. A recent publication claims that this number may be as high as 390 million [8].

There are a number of factors associated with DHF [9], including host-related factors. Low platelet count is known to be a characteristic clinical manifestation in severe dengue, and a platelet count of less than $50,000 / \mathrm{mm}^{3}$ on days 5 to 7 after the onset of illness is associated with the development of complications, such as bleeding and shock $[10,11]$. 
A recent genome-wide association study (GWAS) of hematological and biochemical traits identified six SNPs associated with low platelet count in healthy subjects [12]. These SNPs might also affect susceptibility to severe dengue. In the present study, to elucidate genetic factors in the host that influence the pathogenesis of dengue infection, the association of SNPs involved in low platelet count was examined in patients with dengue.

\section{Methods}

\section{Patients}

A total of 918 Thai patients with dengue who were treated at Ratchaburi Hospital and Lampang Hospital, Thailand, between 1999 and 2004 were investigated. All patients were $\leq 15$ years old at diagnosis. They were confirmed as having DEN infection by dengue IgM/IgG capture enzyme-linked immunosorbent assay (ELISA), reverse transcriptase polymerase chain reaction (RTPCR), and/or DEN isolation at the Arbovirus Laboratory, National Institute of Health, Department of Medical Sciences, Ministry of Public Health, Thailand. The patients were classified into two groups, DHF and DF, according to the WHO 1997 criteria [3]. In this study, DSS (i.e., DHF grades III and IV) is regarded as DHF. In brief, DF was defined as an acute illness with various nonspecific symptoms (e.g., headache, myalgia, arthralgia, rashes, and leucopenia). Patients with DHF had hemorrhagic manifestations, plasma leakage, and thrombocytopenia. Thrombocytopenia was diagnosed by platelet count of less than 100000 per $\mathrm{mm}^{3}$ and a greater than $20 \%$ increase in the packed cell volume from the baseline.

\section{Genotyping}

Genomic DNA was extracted from peripheral blood leukocytes using the QIAamp Blood Kit (Qiagen, Hilden, Germany). Patients with dengue were genotyped for five SNPs, rs5745568 in BAK1, rs6141 in THPO, rs6065 in GP1BA, rs739496 in SH2B3, and rs385893 in RCL1, using TaqMan SNP genotyping assays (Applied Biosystems, Foster City, CA, USA). These SNPs were reported to be associated with low platelet count in a recent GWAS [12]. Although rs7775698 (HBS1L) also showed a significant association with low platelet count [12], it was not analyzed in the present study because the TaqMan probe was not available. In addition, rs4804803 in $C D 209$ was also genotyped to examine the possible interaction with rs5745568 in $B A K 1$.

\section{Statistical analysis}

The deviation from Hardy-Weinberg equilibrium was examined in both DHF and DF groups using a web tool [13]. To assess the association of each SNP with severe dengue, allele frequencies were compared using a Chisquare test between the two groups. The allelic odds ratio (OR) and the $95 \%$ confidence interval $(95 \% \mathrm{CI}$ ) were calculated for the allele that was previously shown to be associated with low platelet count [12], which allowed us to examine the consistency in the direction of association, because low platelet count is likely to increase the risk for DHF in patients with dengue. Dominant and recessive models were examined for rs5745568 in $B A K 1$. To control possible confounding factors, such as age, sex, hospital (i.e., geographic region), immune status (i.e., primary or secondary infection), and virus serotype, a logistic regression analysis adjusted for these independent variables was performed. In this analysis, the number of risk alleles (i.e., 0, 1, and 2) was used as an independent variable. The significance level was set at $P<0.05$. SNPinfo [14] was used for in silico predictions of functional significance and for detection of SNPs in linkage disequilibrium (LD) with $\operatorname{rs} 5745568\left(r^{2} \geq 0.8\right)$ in the HapMap-CHB and HapMap-JPT populations $[15,16]$.

\section{Results and discussion}

A total of 918 confirmed patients with dengue, including 509 patients with DHF and 409 patients with DF, were investigated in this study (Table 1). They were genotyped for six SNPs. Of which, five SNP, rs5745568 in BAK1, rs6141 in THPO, rs6065 in GP1BA, rs739496 in SH2B3, and rs385893 in $R C L 1$, were previously reported to be associated with low platelet count [12]. Although rs7775698 (HBS1L) also showed a significant association with low platelet count in a previous GWAS [12], it was not analyzed in the present study because rs7775698 could not be successfully genotyped. The genotype and allele frequencies of the SNPs in each dengue group are

Table 1 Patient characteristics (total $n=918$ )

\begin{tabular}{lll}
\hline Characteristic & DHF $n=509$ & DF $n=409$ \\
\hline Sex & 276 & 213 \\
Male & 233 & 196 \\
Female & & \\
Hospital & 134 & 172 \\
Lampang & 375 & 237 \\
Ratchaburi & & \\
Immune status & 65 & 94 \\
Primary & 444 & 314 \\
Secondary & & 153 \\
Serotype of virus & 151 & 109 \\
D1 & 197 & 92 \\
D2 & 79 & 54 \\
D3 & 81 & $9.0(1-15)$ \\
D4 & $10(0-15)$ & \\
Age ${ }^{\text {a (years) }}$
\end{tabular}

${ }^{a}$ Median (minimum-maximum) 
shown in Table 2. No SNP showed a significant deviation from the Hardy-Weinberg equilibrium in either dengue group. Among SNPs examined in this study, rs5745568 in $B K A 1$ was the only SNP that was significantly associated with a risk for DHF $[P=0.006$ and crude OR $(95 \% \mathrm{CI})=1.32(1.09-1.60)]$. A further analysis based on the genotype revealed that the GG genotype of rs5745568 significantly increased a risk for DHF compared to GT and TT $[P=0.003$ and crude OR (95\% $\mathrm{CI})=1.50(1.15-1.95)]$, while GG and GT did not significantly increase a risk compared to TT $[P=0.279$ and crude OR $(95 \% \mathrm{CI})=1.26(0.83-1.93)]$, indicating that a recessive model best explains the association of $G$ allele of rs5745568 with DHF. The association of rs5745568-G with DHF was also significant in a logistic regression analysis adjusted for age, sex, hospital, immune status, and virus serotype $[P=0.016$ and adjusted $\mathrm{OR}(95 \% \mathrm{CI})=1.29$ $(1.05-1.58)]$. The other SNPs showed no significant association in the logistic regression analyses (Table 2).
Several studies have shown significant association of a CD209 promoter SNP (rs4804803) with DF or DHF [17-19]. To examine if a CD209 promoter SNP affects the association of $B A K 1 \mathrm{SNP}$ with DHF, we analyzed dengue patients for rs4804803. The rs4804803 SNP was not significantly associated with DHF in this study (Table 2). A logistic regression analysis adjusted for age, sex, hospital, immune status, virus serotype, and genotype of rs4804803 revealed that rs5745568 was significantly associated with DHF $[P=0.0167$ and adjusted OR $(95 \% \mathrm{CI})=1.29(1.05-1.58)]$. Thus, we conclude that the association of rs5745568 with DHF is independent of rs4804803.

Although the direct association of rs5745568-G with low platelet count in patients with dengue was not examined in this study, rs5745568-G has been reported to be associated with low platelet count in healthy subjects [12]. Together with our results, the low-level constitutive production of platelets caused by the $\mathrm{G}$ allele of rs 5745568

Table 2 Association of SNPs with DHF

\begin{tabular}{|c|c|c|c|c|c|c|c|}
\hline \multirow[t]{2}{*}{ SNP ID (gene) } & \multirow{2}{*}{$\begin{array}{l}\text { Genotype } \\
\text { (Allele) }\end{array}$} & \multicolumn{2}{|c|}{ Patients with dengue } & \multicolumn{2}{|l|}{ Chi-square test } & \multicolumn{2}{|c|}{ Logistic regression analysis } \\
\hline & & $\overline{\mathrm{DHF}}$ & DF & Crude OR (95 \% Cl) & $P$-value & Adjusted OR (95 \% Cl) & $P$-value \\
\hline \multirow[t]{4}{*}{ rs5745568 (BAK1) } & $\pi$ & 48 & 47 & & & & \\
\hline & GT & 191 & 187 & & & & \\
\hline & GG & 265 & 173 & & & & \\
\hline & G-allele & $721(71.5 \%)$ & $533(65.5 \%)$ & $1.32(1.09-1.60)$ & 0.006 & $1.29(1.05-1.58)$ & 0.016 \\
\hline \multirow[t]{4}{*}{ rs6141 (THPO) } & $\pi$ & 82 & 70 & & & & \\
\hline & $\mathrm{CT}$ & 260 & 189 & & & & \\
\hline & $\mathrm{CC}$ & 164 & 148 & & & & \\
\hline & C-allele & $588(58.1 \%)$ & $485(59.6 \%)$ & $0.94(0.78-1.14)$ & 0.520 & $0.98(0.80-1.19)$ & 0.844 \\
\hline \multirow[t]{4}{*}{ rs6065 (GP1BA) } & $\pi$ & 2 & 0 & & & & \\
\hline & $\mathrm{CT}$ & 37 & 42 & & & & \\
\hline & $\mathrm{CC}$ & 467 & 366 & & & & \\
\hline & C-allele & 971 (96.0 \%) & 774 (94.9 \%) & $1.29(0.83-2.00)$ & 0.263 & $1.35(0.85-2.14)$ & 0.203 \\
\hline \multirow[t]{4}{*}{ rs739496 (SH2B3) } & AA & 18 & 12 & & & & \\
\hline & $A G$ & 137 & 114 & & & & \\
\hline & GG & 350 & 281 & & & & \\
\hline & A-allele & $173(17.1 \%)$ & 138 (17.0 \%) & $1.01(0.79-1.29)$ & 0.921 & $0.96(0.74-1.24)$ & 0.731 \\
\hline \multirow[t]{4}{*}{ rs385893 (RCL1) } & $\pi$ & 49 & 39 & & & & \\
\hline & $\mathrm{CT}$ & 200 & 165 & & & & \\
\hline & $\mathrm{CC}$ & 258 & 205 & & & & \\
\hline & T-allele & 298 (29.4 \%) & 243 (29.7 \%) & $0.98(0.80-1.21)$ & 0.882 & $1.02(0.83-1.25)$ & 0.886 \\
\hline \multirow[t]{4}{*}{ rs4804803 (CD209) } & AA & 411 & 323 & & & & \\
\hline & GA & 92 & 77 & & & & \\
\hline & GG & 4 & 8 & & & & \\
\hline & G-allele & 100 (9.8 \%) & 93 (11.4\%) & $0.85(0.63-1.15)$ & 0.288 & $0.83(0.61-1.13)$ & 0.242 \\
\hline
\end{tabular}

Frequencies are shown in parentheses (\%). The odds ratio (OR) of rs5745568 in BAK1, rs6141 in THPO, rs6065 in GP1BA, rs739496 in SH2B3, and rs385893 in RCL1 was calculated for the allele associated with low platelet count in a previous GWAS [12]. The OR of rs4804803 in CD209 was calculated for the G allele. Genotype frequencies at SNPs examined did not deviate from those expected from the Hardy-Weinberg equilibrium in DHF and DF groups 
may increase the risk of bleeding in dengue infection. To fully understand the biological significance of rs5745568 in the pathogenesis of dengue infection, the difference in the time course of the platelet count among dengue patients with different genotypes requires to be studied in future.

The rs5745568 SNP is located upstream of BAK1. To estimate the functional significance of rs5745568, an in silico analysis [14] was performed. The rs5745568 was predicted to be located in the putative binding sites of various transcription factors. Although the expression level of $B A K 1$ remains to be studied in patients with dengue, the $B A K 1$ expression level may vary among genotypes of rs5745568. At this locus, a total of eight SNPs were in LD $\left(r^{2} \geq 0.8\right)$ with rs5745568 in the HapMapCHB or HapMap-JPT population (data not shown), which raises the possibility that the significant association of the $\mathrm{G}$ allele of rs 5745568 with a risk for DHF may be explained by another causative variant in LD with rs5745568. Thus, it should be noted that rs5745568 may not be primarily associated with DHF.

BCL-2 homologous antagonist/killer (BAK), encoded by $B A K 1$, is a member of the BCL-2 family of apoptosis regulators, including pro-apoptotic (BAX, BAD, BAK, and $\mathrm{BOK}$ ) and anti-apoptotic (BCL-2, BCL-XL, and $\mathrm{BCL}-\mathrm{W})$ proteins. Bak is responsible for mediating platelet death in mice [20], which implies that the G allele of rs5745568 may be associated with a higher expression level of BAK in patients with dengue as well as in healthy individuals. The present results suggest that BAK plays a crucial role in the pathogenesis of DHF and is a potential target of therapy for DHF.

\section{Conclusions}

The $\mathrm{G}$ allele of rs5745568 in $B A K 1$ was significantly associated with a risk for DHF. Although the association of the $\mathrm{G}$ allele of rs5745568 with low platelet count in patients with dengue remains to be confirmed in future studies, the present results suggest that the BAK protein, encoded by $B A K 1$, plays a crucial role in the pathogenesis of DHF.

\footnotetext{
Acknowledgments

We sincerely thank patients who participated in this study. The authors are grateful to Dr Chuan-Liang Kao and Dr Kalichamy Alagarasu for their valuable comments and suggestions that improved the manuscript. This work was partly supported by KAKENHI (Grant-in-Aid for Scientific Research) on Priority Areas "Applied Genomics" from the Ministry of Education, Culture, Sports, Science and Technology of Japan.
}

\section{Authors' contributions}

TND and JO wrote the manuscript. TND performed statistical analyses and genotyping. AS and IN extracted DNA. AS, SA, PS, and JP collected blood samples and contributed to the acquisition of clinical data. TND, JP, and JO participated in the design and coordination of the study. NT was involved in the interpretation of the data and preparation of the manuscript. All authors read and approved the final manuscript.

\section{Competing interests}

The authors declare that they have no competing interests.

\section{Ethics approval and consent to participate}

This study was approved by the Institute Review Board for Research in Human Subjects, Department of Medical Science, Ministry of Public Health, Thailand; the Institute Review Board of the Faculty of Tropical Medicine, Mahidol University, Thailand; the Research Ethics Committee of the Faculty of Medicine, University of Tsukuba, Japan; and the Research Ethics Committee of the Faculty of Science, The University of Tokyo, Japan. Unlinked anonymous blood samples were obtained from the unidentifiable leftover blood of laboratory diagnosis of dengue infection. According to the guideline of Thailand [21], no specific consent forms were required from the patients in this case, since the Ethics Committee approved.

\section{Author details}

'Graduate School of Comprehensive Human Sciences, University of Tsukuba, Tsukuba, Japan. ${ }^{2}$ Faculty of Public Health, University of Medicine and Pharmacy at Ho Chi Minh city, Ho Chi Minh City, Viet Nam. ${ }^{3}$ Faculty of Medicine, University of Tsukuba, Tsukuba, Japan. ${ }^{4}$ Graduate School of Science, The University of Tokyo, Tokyo, Japan. ${ }^{5}$ Department of Medical Sciences, Ministry of Public Health, Nonthaburi, Thailand. ${ }^{6}$ Food and Drug Administration, Ministry of Public Heath, Nonthaburi, Thailand. ${ }^{7}$ Faculty of Tropical Medicine, Mahidol University, Bangkok, Thailand.

Received: 5 August 2015 Accepted: 22 June 2016

Published online: 11 July 2016

\section{References}

1. Simmons CP, Farrar J, Chau NV, Wills B. Current concepts dengue. New Engl J Med. 2012;366:1423-32.

2. Fried JR, Gibbons RV, Kalayanarooj S, Thomas SJ, Srikiatkhachorn A, Yoon IK, Jarman RG, Green S, Rothman AL,Cummings DA. Serotype-specific differences in the risk of dengue hemorrhagic fever: an analysis of data collected in Bangkok, Thailand from 1994 to 2006. PLoS Negl Trop Dis. 2010;4:e617.

3. Chow VT. Molecular diagnosis and epidemiology of dengue virus infection. Ann Acad Med Singapore. 1997;26:820-6.

4. Rigau-Perez JG, Clark GG, Gubler DJ, Reiter P, Sanders RJ, Vorndam AV. Dengue and dengue haemorrhagic fever. Lancet. 1998;352:971-7.

5. Anders KL, Nguyet NM, Chau NV, Hung NT, Thuy TT, Lien le B, Farrar J, Wills B, Hien TT,Simmons CP. Epidemiological factors associated with dengue shock syndrome and mortality in hospitalized dengue patients in Ho Chi Minh City, Vietnam. Am J Trop Med Hyg. 2011;84:127-34.

6. World Health Organization. Dengue: Guidelines for Diagnosis, Treatment, Prevention and Control: New Edition. http://www.who.int/rpc/guidelines/ 9789241547871/en. Accessed 24 July 2015.

7. Gibbons RV, Vaughn DW. Dengue: an escalating problem. BMJ. 2002;324:1563-6.

8. Bhatt S, Gething PW, Brady OJ, Messina JP, Farlow AW, Moyes CL, Drake JM, Brownstein JS, Hoen AG, Sankoh O, Myers MF, George DB, Jaenisch T, Wint GR, Simmons CP, Scott TW, Farrar JJ,Hay SI. The global distribution and burden of dengue. Nature. 2013;496:504-7.

9. Huy NT, Van Giang T, Thuy DH, Kikuchi M, Hien TT, Zamora J, Hirayama K. Factors associated with dengue shock syndrome: a systematic review and meta-analysis. PLoS Negl Trop Dis. 2013;7:e2412.

10. Malavige GN, Velathanthiri VGNS, Wijewickrama ES, Fernando S, Jayaratne SD, Aaskov J, Seneviratne SL. Patterns of disease among adults hospitalized with dengue infections. Qjm-an Int J Med. 2006;99:299-305.

11. Hammond SN, Balmaseda A, Perez L, Tellez Y, Saborio SI, Mercado JC, Videa E, Rodriguez Y, Perez MA, Cuadra R, Solano S, Rocha J, Idiaquez W, Gonzalez A, Harris E. Differences in dengue severity in infants, children, and adults in a 3-year hospital-based study in Nicaragua. Am J Trop Med Hyg. 2005; $73: 1063-70$

12. Kamatani Y, Matsuda K, Okada Y, Kubo M, Hosono N, Daigo Y, Nakamura Y, Kamatani N. Genome-wide association study of hematological and biochemical traits in a Japanese population. Nat Genet. 2010;42:210-5.

13. Rodriguez S, Gaunt TR, Day IN. Hardy-Weinberg equilibrium testing of biological ascertainment for Mendelian randomization studies. Am J Epidemiol. 2009;169:505-14. 
14. Xu Z, Taylor JA. SNPinfo: integrating GWAS and candidate gene information into functional SNP selection for genetic association studies. Nucleic Acids Res. 2009;37:W600-5.

15. The_International HapMap Consortium. The International HapMap Project. Nature. 2003;426:789-96.

16. The_International HapMap Consortium. A haplotype map of the human genome. Nature. 2005;437:1299-320.

17. Sakuntabhai A, Turbpaiboon C, Casadémont I, Chuansumrit A, Lowhnoo T, Kajaste-Rudnitski A, Kalayanarooj SM, Tangnararatchakit K,Tangthawornchaikul N, Vasanawathana S, Chaiyaratana W, Yenchitsomanus PT, Suriyaphol P, Avirutnan P, Chokephaibulkit K, Matsuda F,Yoksan S, Jacob Y, Lathrop GM, Malasit P, Desprès P, Julier C. A variant in the CD209 promoter is associated with severity of dengue disease. Nat Genet. 2005;37:507-13.

18. Xavier-Carvalho C, Gibson G, Brasil P, Ferreira RX, de Souza SR, Gonçalves Cruz O, de Oliveira SA, de Sá Carvalho M, Pacheco AG, Kubelka CF, Moraes MO. Single nucleotide polymorphisms in candidate genes and dengue severity in children: a case-control, functional and meta-analysis study. Infect Genet Evol. 2013;20:197-205.

19. Wang L, Chen RF, Liu JW, Lee IK, Lee CP, Kuo HC, Huang SK, Yang KD. DC SIGN (CD209) Promoter -336 A/G polymorphism is associated with dengue hemorrhagic fever and correlated to DC-SIGN expression and immune augmentation. PLoS Negl Trop Dis. 2011:5:e934.

20. Mason KD, Carpinelli MR, Fletcher Jl, Collinge JE, Hilton AA, Ellis S, Kelly PN, Ekert PG, Metcalf D, Roberts AW, Huang DC, Kile BT. Programmed anuclear cell death delimits platelet life span. Cell. 2007;128:1173-86.

21. Review of the Informed Consent. [http://www.tm.mahidol.ac.th/research/ Ethics/human/sop/ECS-006-04.pdf]. Accessed 24 July 2015.

\section{Submit your next manuscript to BioMed Central and we will help you at every step:}

- We accept pre-submission inquiries

- Our selector tool helps you to find the most relevant journal

- We provide round the clock customer support

- Convenient online submission

- Thorough peer review

- Inclusion in PubMed and all major indexing services

- Maximum visibility for your research

Submit your manuscript at www.biomedcentral.com/submit
Biomed Central 